\title{
Acute kidney injury after Fontan completion: Risk factors and outcomes
}

Jesse J. Esch, MD, ${ }^{\mathrm{a}}$ Joshua M. Salvin, MD, MPH, ${ }^{a}$ Ravi R. Thiagarajan, MD, MPH, ${ }^{a}$

Pedro J. del Nido, MD, ${ }^{b}$ and Satish K. Rajagopal, $\mathrm{MD}^{\mathrm{a}}$

\section{ABSTRACT}

Objective: Acute kidney injury (AKI) is a predictor of outcomes in heterogeneous populations of children undergoing cardiac surgery. We investigated its causes and consequences in a cohort undergoing Fontan completion, hypothesizing that central venous pressure is independently associated with development of AKI.

Methods: In this retrospective cohort study of patients undergoing Fontan ( $\mathrm{n}=211)$, univariable and multivariable analyses identified factors associated with AKI within 3 days of surgery. Secondary analyses identified factors associated with hospital length of stay, and examined effects of perioperative kidney injury on follow-up renal function.

Results: Acute kidney injury occurred in $42 \%$ of cases $(n=89)$, with the following independent risk factors: mean renal perfusion (mean arterial minus central venous) pressure on postoperative day zero (per $\mathrm{mm} \mathrm{Hg}$; adjusted odds ratio [AOR] $0.83 ; P<.001)$; preoperative atrioventricular valve regurgitation $>$ mild (AOR 6.78; $P=.02$ ); bypass time (per 10 minutes, AOR 1.08; $P=.04$ ); peak inotrope score on postoperative day zero (per point, AOR 1.17; $P<.001$ ); and preoperative pulmonary vascular resistance (per Wood unit, AOR $1.69 ; P=.04)$. Central venous pressure was not independently associated with AKI. Moderate and severe (but not mild) AKI were independently associated with prolonged hospital length of stay (adjusted hazard ratios, $0.56 ; P=.004$, and $.41 ; P=.006$, respectively). Perioperative injury was not associated with longer-term renal dysfunction.

Conclusions: Acute kidney injury is common after Fontan completion and has several potentially modifiable risk factors. Moderate-to-severe injury is associated with longer hospital length of stay but not with renal dysfunction at follow-up. (J Thorac Cardiovasc Surg 2015;150:190-7)

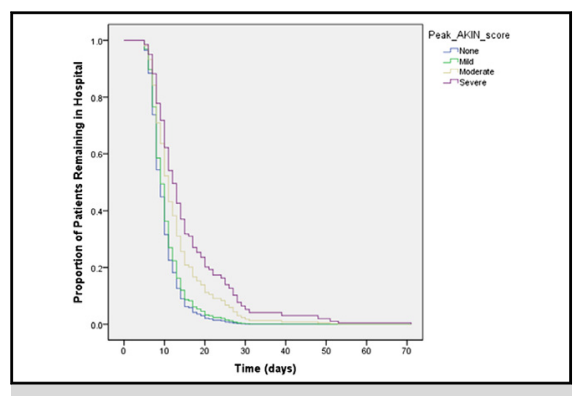

Effect of acute kidney injury on length of stay after Fontan completion.

\section{Central Message}

Acute kidney injury is common after Fontan completion, and moderate-to-severe AKI predicts longer hospital length of stay. Injury seems to be mediated more by systemic hypotension than by central venous hypertension. Maintaining postoperative renal perfusion pressure while moderating inotrope use might reduce risk of injury and thus length of stay.

\section{Perspective}

Acute kidney injury in adults undergoing cardiac surgery is strongly associated with adverse outcomes. The importance of cardiac surgery-associated AKI in children is less clear. Patients undergoing Fontan completion face an acute rise in renal venous pressure and thus comprise an appealing model population. Acute kidney injury is common after the Fontan procedure, and moderate-to-severe AKI predicts longer hospital length of stay. Lower renal perfusion pressure and higher peak inotrope score are independent risk factors for AKI, whereas higher central venous pressure is not. This study provides important insight on the pathophysiology of AKI, and suggests strategies to mitigate $\mathrm{AKI}$ in this population.

See Editorial Commentary page 197.
From the a Department of Cardiology, Boston Children's Hospital; and ${ }^{\mathrm{b}}$ Department of Cardiovascular Surgery, Harvard Medical School, Boston, Mass.

This research project received support from the Tommy Kaplan Cardiovascular Fellowship Fund (salary support for Dr Esch).

Received for publication Nov 11, 2014; revisions received March 23, 2015; accepted for publication April 2, 2015; available ahead of print May 9, 2015.

Address for reprints: Satish K. Rajagopal, MD, Department of Cardiology, 300 Longwood Ave, Boston, MA 02115 (E-mail: Satish.Rajagopal@ cardio.chboston.org). 0022-5223/\$36.00

Copyright (c) 2015 by The American Association for Thoracic Surgery

http://dx.doi.org/10.1016/j.jtcvs.2015.04.011
Acute kidney injury (AKI) in adults undergoing cardiac surgery is strongly associated with adverse outcomes, including death, and the development of chronic kidney disease (CKD). ${ }^{1,2}$ The importance of cardiac surgeryassociated AKI in children is more controversial, and the epidemiology is not as well characterized, a fact reflected by the wide range of reported ${ }^{3,4}$ incidences $(15 \%-52 \%)$. Available evidence in children suggests that moderate to 


\section{Abbreviations and Acronyms \\ AHR $=$ adjusted hazard ratio \\ $\mathrm{AKI}=$ acute kidney injury \\ $\mathrm{AOR}=$ adjusted odds ratio \\ $\mathrm{CAP}=$ common atrial pressure \\ $\mathrm{CI}=$ confidence interval \\ $\mathrm{CKD}=$ chronic kidney disease \\ $\mathrm{IQR}=$ interquartile range \\ $\mathrm{MAP}=$ mean arterial pressure \\ $\mathrm{POD}=$ postoperative day}

severe AKI prolongs mechanical ventilation time, need for inotropic support, and lengths of stay in both the intensive care unit and hospital, ${ }^{3-6}$ although the implications for mortality and development of CKD are less clear. ${ }^{3,7}$ Whether risk factors for cardiac surgery-associated AKI in children are modifiable, and whether preventing AKI would result in improved outcomes, remain open questions.

Several investigators have begun to explore risk factors for the development of AKI and its implications for children undergoing congenital heart surgery ${ }^{3,4,8}$; data are sparse regarding the relationship between specific surgical procedures and AKI. Patients undergoing Fontan completion comprise an appealing study population from which to improve our understanding of AKI. Incorporation of the inferior vena cava into the pulmonary arteries results in an abrupt increase in renal vein pressure that provides a unique window into the effects of systemic venous hypertension on the development of AKI. Moreover, these patients face a significant lifetime risk of developing CKD, the presence of which is an important risk factor for late death and need for cardiac transplantation. ${ }^{10}$

We sought to identify risk factors for the development of AKI in patients undergoing Fontan completion. We hypothesized that higher postoperative Fontan (ie, central venous) pressure is an independent risk factor for AKI. Second, we investigated the implications of AKI for hospital length of stay and longer-term renal function.

\section{METHODS}

This retrospective, single-center cohort study included all patients undergoing Fontan completion at Boston Children's Hospital, between October 2003 and June 2009, in whom a recent (within 30 days) preoperative serum creatinine value was available. These dates were selected based on availability of an electronic medical record with granular intra- and post-operative hemodynamic data, along with a pre-existing Fontan database. A priori exclusion criteria were: (1) Fontan revision rather than completion; (2) Fontan comprising only incorporation of hepatic veins (ie, previous Kawashima operation); (3) dependence on renal replacement therapy at the time of the Fontan procedure; (4) early postoperative extracorporeal membrane oxygenation (within 48 hours) or death (within 30 days); and (5) absence of invasive central venous pressure monitoring on postoperative day (POD) 0 . The protocol was approved by the Boston Children's Hospital Committee on Clinical Investigation.

\section{Data Collection}

Medical records were reviewed for demographic, anatomic, and physiologic details. Baseline creatinine was defined as the most recent serum creatinine level $(\mathrm{mg} / \mathrm{dL})$ within 30 days of Fontan completion. The coefficient of variation for the Roche (Mannheim, Germany) mass spectroscopy-traceable enzymatic creatinine assay used in the Boston Children's Hospital chemistry laboratory was $1.8 \%$ during the study period. Estimated creatinine clearance was calculated using the modified Schwartz and colleagues' formula ${ }^{11}: 0.413 \times$ Height $(\mathrm{cm}) /$ creatinine $(\mathrm{mg} / \mathrm{dL})$. The estimated creatinine clearance was used as a surrogate for glomerular filtration rate, with $80 \mathrm{~mL} /$ minute $/ 1.73 \mathrm{~m}^{2}$ used as the lower limit of normal to define renal dysfunction. ${ }^{12}$

The preoperative data collected comprised echocardiographic indices (degree of atrioventricular valve regurgitation and systemic ventricular dysfunction), hemodynamic data from pre-Fontan catheterization (mean pulmonary artery and atrial pressures, transpulmonary gradient, systemic ventricular end diastolic pressure, pulmonary vascular resistance), and preoperative use of potential nephrotoxins (diuretics, angiotensinconverting enzyme inhibitors, aminoglycoside antibiotics). To consider the potential effects of intravenous contrast agents on kidney function, the time interval between pre-Fontan catheterization and surgery was recorded. Intraoperative data collected comprised type of Fontan (lateral tunnel vs extracardiac), fenestration creation, additional surgical maneuvers performed (eg, valvuloplasty), total cardiopulmonary bypass time, total aortic crossclamp time, lowest intraoperative bypass flow rate, intraoperative urine output, lowest intraoperative hematocrit, lowest intraoperative rectal temperature, peak intraoperative lactate, and use of aprotinin.

Postoperative data were summarized by day: POD0 was defined as the period from postoperative admission to the cardiac intensive care unit until 7:00 PM the following morning; POD1, POD2, and POD3 were defined as sequential 24-hour intervals thereafter. Mean arterial pressure (MAP; mm $\mathrm{Hg}$ ) was analyzed using arterial line data when available and cuff blood pressure once the arterial line was removed. Fontan pressure $(\mathrm{mm} \mathrm{Hg})$ was measured from a central line in the internal jugular vein. Common atrial pressure (CAP; $\mathrm{mm} \mathrm{Hg}$ ) was measured from a transthoracic catheter placed in the operating room. Renal perfusion pressure $(\mathrm{mm} \mathrm{Hg}$ ) at any point in time was defined as MAP minus Fontan pressure. Review of the medical record by the investigators-blinded to AKI status-allowed documentation of a representative MAP, Fontan pressure, CAP, and renal perfusion pressure for each hour of monitoring (median after excluding data points that were clearly "noise"-for example, pressures while infusing through the line). For each POD, median MAP, Fontan pressure, $\mathrm{CAP}$, and renal perfusion pressure were calculated using hourly values. Additionally, daily nadirs of MAP and renal perfusion pressure were recorded, as were daily peak Fontan pressures. Inotrope score was defined as ${ }^{13}$ : [dose of dopamine in $\mathrm{mcg} / \mathrm{kg} /$ minute] + [dose of milrinone in $\mathrm{mcg} / \mathrm{kg} /$ minute] $\times 10+$ [dose of epinephrine in $\mathrm{mcg} / \mathrm{kg} /$ minute $\times 100$. For each POD, peak inotrope score was recorded.

Peak postoperative lactate was defined as the highest lactate measured in the cardiac intensive care unit before the end of POD3. Daily fluid intake, volume of packed red blood cell transfusion, urine output, and total output were indexed to patient weight. Absolute daily fluid balance was recorded. For each day, "diuretic intensity" was determined by counting the number of distinct diuretics used (furosemide, bumetanide, chlorothiazide, spironolactone). Use of nephrotoxins (aminoglycosides, vancomycin, angiotensin-converting enzyme inhibitors, amphotericin) was recorded. Additional postoperative data collected included "significant arrhythmia" (defined as any rhythm other than sinus rhythm or junctional rhythm paced AAI or DDD), use of cooling, and echocardiographic measures of systemic ventricular dysfunction and atrioventricular valve regurgitation (defined using postoperative transesophageal echocardiogram, if available, and otherwise, using predischarge transthoracic echocardiogram). Each patient's most recent serum creatinine (with paired height) was used to 
calculate estimated creatinine clearance as a marker of renal function at last follow-up.

\section{Outcome Variables}

The primary outcome variable was defined as the presence of AKI at any time between PODs 0 and 3. In accordance with the Acute Kidney Injury Network criteria for AKI,${ }^{14}$ the ratio of peak serum creatinine on each POD to preoperative serum creatinine defined the change in renal function. The patient was considered to have: no AKI if the peak ratio remained $<1.5$, or if the absolute change in serum creatinine was $<0.3 \mathrm{mg} / \mathrm{dL}$ (so that a change from $0.2 \mathrm{~g} / \mathrm{dL}$ to $0.4 \mathrm{~g} / \mathrm{dL}$ would not qualify as $\mathrm{AKI}$ ); mild $\mathrm{AKI}$ if the highest ratio attained was 1.5 to 2 ; moderate AKI if the highest ratio was 2 to 3 ; and severe $\mathrm{AKI}$ if the peak ratio was $>3$, or if the patient required renal replacement therapy. Given the controversy regarding the optimal definition of AKI in children, we additionally performed an analysis using the Acute Dialysis Quality Initiative Pediatric Risk-InjuryFailure-Loss (pRIFLE) definition. This defines AKI by percentage decline in estimated creatinine clearance, with risk being a decline of $25 \%$ to $50 \%$; injury, $50 \%$ to $75 \%$; and failure, $>75 \%$ decline. ${ }^{15}$ Urine output was not used as a criterion to define AKI.

\section{Statistical Analysis}

Statistical analysis was performed using IBM SPSS, version 19 (PASW, Inc, Chicago, Ill). Data are reported as frequency (n) with proportion (\%), or median with interquartile range (IQR, 25th-75th percentile). Significance of association with AKI was assessed using $\chi^{2}$ analysis and the Fisher exact test for discrete variables; and the Mann-Whitney $U$ and Kruskal-Wallis tests for continuous variables. Variables associated with AKI to a significance level of $<0.1$ in univariable analysis were entered into a binary logistic regression model, using forward conditional selection given the large number of predictor variables. A correlation matrix of the potential predictor variables revealed that none of the retained variables was multicollinear. Log-rank testing was used to identify variables associated with hospital length of stay and AKI, and those variables associated with both were entered into a multivariable Cox model to identify independent associations.

\section{RESULTS \\ Cohort Characteristics}

A total of 211 patients met the inclusion criteria. Forty percent $(n=85)$ of the cohort was female. Median age and weight at Fontan completion were 2.7 years (IQR: 2.2-3.3 years) and $12.5 \mathrm{~kg}$ (IQR: 11.1-14.2 kg). Most $(88 \% ; \mathrm{n}=186)$ underwent a lateral tunnel-type Fontan and had a fenestration created $(96 \% ; n=202)$. All underwent cardiopulmonary bypass. Sixty-five percent $(n=138)$ of the patients had systemic right ventricles, the majority of whom had hypoplastic left heart syndrome $(50 \% ; n=105)$. Systemic ventricular dysfunction and more than mild atrioventricular valve regurgitation were present in $22 \%(\mathrm{n}=46)$ and $10 \%(\mathrm{n}=20)$ of the cohort, respectively. Median preoperative estimated creatinine clearance was $110 \mathrm{~mL} /$ minute $/ 1.73 \mathrm{~m}^{2}$ (IQR: $90-125 \mathrm{~mL} / \mathrm{minute} / 1.73 \mathrm{~m}^{2}$ ). Baseline estimated creatinine clearance was low in 21 patients $(10 \%)$. Preoperative use of angiotensin-converting enzyme inhibitors $(78 \%)$ and diuretics $(42 \%)$ was common. No patient underwent dialysis during hospitalization for Fontan completion. Seven patients (3\%) died during the follow-up period at a median of 401 days after Fontan completion (IQR: 104 days to 5.1 years).

Exclusions were as follows: 15 patients had no central venous-pressure monitoring documented on POD0. Five patients had no recent serum creatinine. Five patients required extracorporeal membrane oxygenation support within the first 48 postoperative hours, 1 of whom later died (support withdrawn). An additional 1 patient died 12 days postoperatively as an outpatient (late surgical bleeding).

\section{Acute Kidney Injury: Incidence, Severity, and Time Course}

Eighty-nine patients (42\%) developed AKI within 72 hours of Fontan, with mild injury in $14 \%(n=30)$, moderate injury in $22 \%(\mathrm{n}=46)$, and severe injury in $6 \%(\mathrm{n}=13)$. No difference was found in the incidence or distribution of AKI when the pRIFLE criteria were substituted for the Acute Kidney Injury Network definitions. Of patients experiencing AKI, criteria were first met on: POD0 in 10\%; POD1 in 76\%; POD2 in $10 \%$; and POD3 in 3\%. Peak AKI severity occurred on: POD0 in 3\%; POD1 in $70 \%$; POD2 in $19 \%$; and POD3 in $8 \%$.

\section{Factors Associated With Acute Kidney Injury}

Potential risk factors were tested for an association with the development of AKI (Table 1). Those variables associated with the development of AKI at a $P$ value of $\leq .1$ were used to create a multiple logistic regression model of AKI. Potential postoperative hemodynamic predictors were limited to those on POD0, to avoid injury predating exposure. Results of this analysis are presented in Table 2.

Contrary to our hypothesis, higher mean Fontan pressure was not found to be an independent risk factor for AKI, whereas renal perfusion pressure on POD0 was a risk factor (adjusted odds ratio, $0.83 ; 95 \%$ confidence interval [CI] $0.78-0.90 ; P<.001)$. Other variables retained in the final model were: preoperative pulmonary vascular resistance, the presence of more than mild preoperative atrioventricular valve regurgitation; total cardiopulmonary bypass time; and peak inotrope score on POD0. The model performed well, with an area under the receiver-operator curve of 0.87 (95\% CI: 0.81-0.92).

We performed limited subgroup analyses on patients with significant atrioventricular valve regurgitation. Twenty had $>$ mild atrioventricular valve regurgitation preoperatively; those who developed AKI $(\mathrm{n}=16)$ had lower median MAP (57 vs $63 \mathrm{~mm} \mathrm{Hg} ; P=.02$ ) and renal perfusion pressure (42 vs $48 \mathrm{~mm} \mathrm{Hg} ; P=.047$ ) on POD0, compared with those without AKI $(\mathrm{n}=4)$, whereas median Fontan pressure did not differ (16 vs $15 \mathrm{~mm} \mathrm{Hg}$ ). Thirty-five patients underwent atrioventricular valve plasty along with Fontan; those who developed AKI $(n=19)$ had, in addition, lower median MAP (56 vs $61 \mathrm{~mm} \mathrm{Hg}$; 
TABLE 1. Characteristics of patients with and without acute kidney injury after Fontan completion surgery

\begin{tabular}{|c|c|c|c|}
\hline Variables & No AKI & Any AKI & $P$ value \\
\hline Total patients $=211$ & $122(58)$ & $89(42)$ & \\
\hline \multicolumn{4}{|l|}{ Preoperative } \\
\hline Age at surgery $(\mathrm{y})$ & $2.7(2.3-3.3)$ & $2.6(2.2-3.2)$ & .19 \\
\hline Weight at surgery $(\mathrm{kg})$ & $12.8(11.4-14.6)$ & $12.2(10.9-13.6)$ & .02 \\
\hline Gender (female) & $48(39)$ & $37(42)$ & .74 \\
\hline Systemic RV & $73(60)$ & $65(73)$ & .05 \\
\hline HLHS & $55(45)$ & $50(56)$ & .15 \\
\hline AVV regurgitation $>$ mild & $4(3)$ & $16(18)$ & .003 \\
\hline Ventricular dysfunction (any) & $21(17)$ & $25(28)$ & .16 \\
\hline Preoperative ACEi use & $97(81)$ & $68(76)$ & .44 \\
\hline Preoperative diuretic use & $43(36)$ & $46(52)$ & .02 \\
\hline Catheterization within $72 \mathrm{~h}$ & $22(18)$ & $21(24)$ & .3 \\
\hline $\mathrm{PAP}$ at catheterization $(\mathrm{mm} \mathrm{Hg})$ & $12(10-14)$ & $13(11-15)$ & .07 \\
\hline $\mathrm{CAP}$ at catheterization $(\mathrm{mm} \mathrm{Hg})$ & $8(6-10)$ & $8(7-10)$ & .87 \\
\hline PVR at catheterization (iWU) & $1.6(1.3-2.0)$ & $1.9(1.5-2.3)$ & $<.001$ \\
\hline Estimated baseline creatinine clearance $\left(\mathrm{mL} / \mathrm{min} / 1.73 \mathrm{~m}^{2}\right)$ & $107(88-124)$ & $116(93-128)$ & .12 \\
\hline Low baseline creatinine clearance & $14(11)$ & $7(8)$ & .49 \\
\hline \multicolumn{4}{|l|}{ Operative variables } \\
\hline Lateral tunnel (vs extracardiac) & $106(87)$ & $80(90)$ & .51 \\
\hline Fenestration created & $114(93)$ & $88(99)$ & .06 \\
\hline AVV plasty performed & $16(13)$ & $19(21)$ & .11 \\
\hline Total bypass time $(\mathrm{min})$ & $99(81-116)$ & $116(95-140)$ & $<.001$ \\
\hline Aortic crossclamp time (min) & $46(10-61)$ & $54(0-71)$ & .71 \\
\hline Nadir hematocrit $(\%)$ & $28(27-30)$ & $29(27-30)$ & .28 \\
\hline Circulatory arrest performed & $1(0.8)$ & $4(4.5)$ & .08 \\
\hline \multicolumn{4}{|l|}{ Postoperative variables } \\
\hline Mean MAP POD0 (mm Hg) & $63(60-67)$ & $58(53-62)$ & $<.001$ \\
\hline Nadir MAP POD0 (mm Hg) & $50(47-54)$ & $46(42-50)$ & $<.001$ \\
\hline Mean FontanP POD0 (mm Hg) & $14.5(12.8-15.8)$ & $15.5(13.7-17.3)$ & $<.001$ \\
\hline Peak FontanP POD0 (mm Hg) & $20(17-22)$ & $20(18-22)$ & .14 \\
\hline Mean RPP POD 0 (mm Hg) & $49(46-53)$ & $42(37-46)$ & $<.001$ \\
\hline Mean CAP POD0 (mm Hg) & $7.6(6.1-8.9)$ & $8.3(6.7-10.0)$ & .02 \\
\hline Peak inotrope score POD0 & $8(5-10)$ & $10(7.5-13.5)$ & $<.001$ \\
\hline Active cooling on POD0 & $3(2)$ & $13(15)$ & .001 \\
\hline Significant arrhythmia & $13(11)$ & $17(19)$ & .08 \\
\hline Treated with Lasix infusion & $13(11)$ & $34(38)$ & $<.001$ \\
\hline Estimated CI POD0 $\left(\mathrm{mL} / \mathrm{min} / \mathrm{m}^{2}\right)$ & $3.3(2.8-3.8)$ & $2.9(2.4-3.5)$ & .01 \\
\hline Total intake POD0 $(\mathrm{mL} / \mathrm{kg} / \mathrm{h})$ & $5.7(4.5-7.3)$ & $7.4(5.9-9.6)$ & $<.001$ \\
\hline Total output POD0 (mL/kg/h) & $3.5(2.6-4.5)$ & $3.1(2.4-5.2)$ & .64 \\
\hline PRBC transfused POD0 $(\mathrm{mL} / \mathrm{kg})$ & $2.4(0-14.2)$ & $6.9(0-14.9)$ & .14 \\
\hline Urine output POD0 $(\mathrm{mL} / \mathrm{kg} / \mathrm{h})$ & $1.5(1.1-2.2)$ & $1.1(0.9-1.4)$ & $<.0001$ \\
\hline Fluid balance POD0 $(\mathrm{mL})$ & $456(206-670)$ & $724(393-1020)$ & $<.0001$ \\
\hline Any diuretic use POD0 & $41(34)$ & $20(22)$ & .08 \\
\hline Any nephrotoxin POD0 & $18(15)$ & $7(8)$ & .13 \\
\hline Peak lactate (mmol/L) & $2.1(1.6-2.9)$ & $3.2(2.1-4.7)$ & $<.001$ \\
\hline \multicolumn{4}{|l|}{ Follow-up } \\
\hline Hospital LOS (d) & $8(7-10)$ & $12(9-17)$ & $<.001$ \\
\hline Intensive care LOS (d) & $3(2-4)$ & $5(3-8.5)$ & $<.001$ \\
\hline Chest tube duration $(\mathrm{d})$ & $4(3-5)$ & $6(5-9)$ & $<.001$ \\
\hline Estimated creatinine clearance at follow-up $\left(\mathrm{mL} / \mathrm{min} / 1.73 \mathrm{~m}^{2}\right)$ & $113(93-131)$ & $107(95-125)$ & .48 \\
\hline Low follow-up creatinine clearance & $6(4)$ & $9(11)$ & .18 \\
\hline
\end{tabular}

Discrete variables are reported as frequency (\%); continuous variables are reported as median (interquartile range). $A K I$, Acute kidney injury; $R V$, right ventricle; $H L H S$, hypoplastic left heart syndrome; $A V V$, atrioventricular valve; $A C E i$, angiotensin-converting enzyme inhibitor; $P A P$, pulmonary artery pressure; $C A P$, common atrial pressure; $P V R$, pulmonary vascular resistance; $M A P$, mean systemic arterial pressure; Fontan $P$, Fontan baffle/central venous pressure; $R P P$, renal perfusion pressure; $C I$, cardiac index; $P R B C$, packed red blood cells; $P O D$, postoperative day; $L O S$, length of stay. 
TABLE 2. Multivariable model of acute kidney injury after Fontan completion

\begin{tabular}{lccc}
\hline \multicolumn{1}{c}{ Predictor } & Adjusted odds ratio & 95\% confidence interval & $\boldsymbol{P}$ value \\
\hline Preoperative pulmonary vascular resistance (per Wood unit) & 1.69 & $(1.02-2.78)$ & .04 \\
Preoperative systemic AVVR > mild & 6.78 & $(1.30-35.34)$ & .02 \\
Total bypass time (per 10 min) & 1.08 & $(1.003-1.17)$ & .04 \\
Mean renal perfusion pressure POD0 (per 1 mm Hg) & 0.83 & $(0.78-0.90)$ & $(1.05-1.30)$ \\
Peak inotrope score POD0 & 1.17 & .001 \\
\hline
\end{tabular}

Variables not retained in model: weight, systemic right ventricle, preoperative diuretics, preoperative pulmonary artery pressure, mean arterial pressure POD0, nadir mean arterial pressure POD0, mean Fontan pressure POD0, mean common atrial pressure POD0, fenestration created during surgery, cooled on POD0, rhythm disturbance requiring intervention POD0, diuretics used POD0, total fluid administration POD0 (mL/kg/h), net fluid balance POD0, peak postoperative lactate concentration. Area under receiver-operator curve: $0.87(0.81,0.92)$. $A V V R$, Atrioventricular valve regurgitation; $P O D$, postoperative day.

$P=.016)$ and renal perfusion pressure (40 vs $46 \mathrm{~mm} \mathrm{Hg}$; $P=.012)$, compared with those without AKI $(\mathrm{n}=16)$, whereas median Fontan pressure was comparable (15 $\mathrm{mm} \mathrm{Hg}$ in both groups).

\section{The Effects of Acute Kidney Injury on Outcomes}

In univariable analysis, development of AKI after Fontan completion was associated with: duration of pleural effusions requiring chest tubes (6 vs 4 days; $P<.001$ ); intensive care unit length of stay ( 5 vs 3 days; $P<.001$ ); and hospital length of stay (12 vs 8 days; $P<.001)$. The results of a multivariable Cox analysis of the independent effect of AKI on hospital length of stay are presented in Table 3 and Figure 1. Development of moderate or severe AKI was strongly associated with hospital length of stay (adjusted hazard ratio [AHR], 0.56 ; 95\% CI: $0.38-0.83$; $P=.004$; and AHR, 0.41; 95\% CI: 0.22-0.78; $P=.006$, respectively), whereas mild AKI was not (AHR, 0.88; 95\% CI: $0.58-1.33 ; P=.54)$.

Additional variables found to be independently associated with hospital length of stay were: (1) total cardiopulmonary bypass time (per 10 minutes; AHR, 0.96; 95\% CI: 0.93-0.99; $P=.003$ ); (2) average rate of volume administration on POD0 (per $\mathrm{mL} / \mathrm{kg} / \mathrm{hour}$; AHR, $0.90 ; 95 \%$ CI: $0.85-0.96 ; P=.001$ ); and (3) mean preoperative pulmonary artery pressure (per $\mathrm{mm} \mathrm{Hg}$; AHR, 0.96; 95\% CI: 0.92-1.00; $P=.05$ ). In subgroup analysis, we confirmed that when atrioventricular valve plasty was performed along with Fontan, higher renal perfusion pressure remained associated with shorter hospital length of stay $(P=.025)$.

\section{Short-to Medium-Term Renal Function Follow-up After Fontan Completion}

At least 1 follow-up creatinine was available for 198 patients $(94 \%)$. The median interval between Fontan completion and final available creatinine was 373 days (IQR: 9 days to 3.8 years). The median estimated creatinine clearance of the cohort at follow-up was $111 \mathrm{~mL} /$ minute $/ 1.73 \mathrm{~m}^{2}$ (IQR: $93-130 \mathrm{~mL} /$ minute $/ 1.73$ $\left.\mathrm{m}^{2}\right)$ with $8 \%(\mathrm{n}=16)$ having renal dysfunction (estimated creatinine clearance $<80 \mathrm{~mL} /$ minute $/ 1.73 \mathrm{~m}^{2}$ ). Four of the
16 children $(25 \%)$ with renal dysfunction at follow-up had pre-Fontan renal dysfunction; this association was statistically significant (Fisher exact test $P=.017$; linear-by-linear association: .05). Renal dysfunction at follow-up was not found to be associated with presence or degree of postoperative AKI (Fisher exact test $P=.29$, and linear-by-linear association $P=.30$ ).

\section{DISCUSSION}

This study demonstrates that AKI occurs frequently in the 72-hour postoperative period after Fontan completion, with an overall prevalence of $42 \%$. The degree of AKI is moderate or severe in approximately two thirds of those affected. In most cases, both initial and maximal AKI are apparent by the end of the first POD and will resolve by POD3. We identified 3 potentially modifiable risk factors for the development of AKI in this cohort: renal perfusion pressure on POD0; peak inotrope score on POD0; and time on cardiopulmonary bypass.

Contrary to our hypothesis, no independent relationship was found between central venous pressure and the development of AKI, once the renal perfusion pressure

TABLE 3. Proportional hazards model of hospital length of stay after Fontan completion $(n=210)$

\begin{tabular}{lcc}
\hline \multicolumn{1}{c}{ Predictor variables } & Adjusted HR $(\mathbf{9 5} \% \mathbf{C I})$ & $\boldsymbol{P}$ value \\
\hline $\begin{array}{l}\text { PAP at preoperative catheterization } \\
\text { (mm Hg) }\end{array}$ & $0.96(0.92-1.00)$ & .05 \\
Total bypass time (per $10 \mathrm{~min})$ & $0.96(0.93-0.99)$ & .003 \\
Total intake POD0 (mL/kg/h) & $0.90(0.85-0.96)$ & .001 \\
AKI (reference: no AKI) & & .006 \\
$\quad$ Mild & $0.88(0.58-1.33)$ & .542 \\
Moderate & $0.56(0.38-0.83)$ & .004 \\
Severe & $0.41(0.22-0.78)$ & .006 \\
\hline
\end{tabular}

An HR $<1$ indicates a greater likelihood of remaining hospitalized on any given day. Variables not retained in the model: systemic right ventricle, preoperative pulmonary vascular resistance, median mean arterial pressure POD0, nadir mean arterial pressure POD0, median Fontan pressure POD0, median common atrial pressure POD0, peak inotrope score POD0, induced hypothermia POD0, postoperative arrhythmia, Lasix infusion on POD0, mean renal perfusion pressure POD0, nadir renal perfusion pressure POD0, fluid balance POD0, peak postoperative lactate concentration, diuretics used POD0, exposure to postoperative nephrotoxins. $H R$, Hazard ratio; $C I$, confidence interval; $P A P$, mean pulmonary artery pressure; $P O D$, postoperative day; $A K I$, acute kidney injury. 


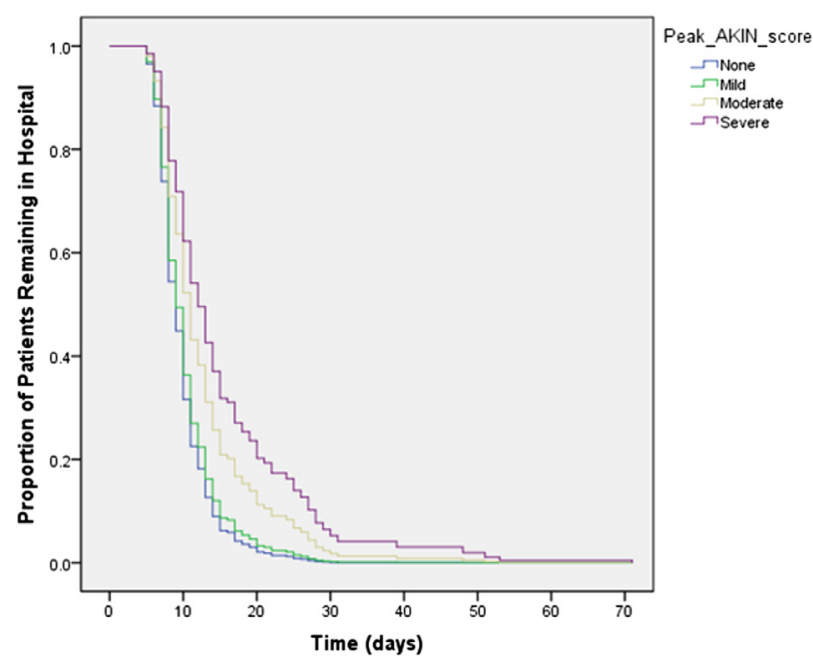

FIGURE 1. Kaplan-Meier curve for hospital length of stay after Fontan completion, stratified by degree of acute kidney injury. AKIN, Acute Kidney Injury Network.

had been taken into account. Moderate-to-severe AKI after Fontan completion has an important independent effect on outcome, with those who develop it being $65 \%$ and $71 \%$ more likely (respectively) to remain hospitalized on any given day than similar patients who do not develop perioperative AKI. Patients who were limited to the development of mild AKI after surgery were not distinguishable from those without AKI. Although moderate-to-severe AKI affects short-term outcomes, it does not seem to have lasting renal consequences.

Although the prevalence of AKI in this cohort of patients is similar to that in those from other studies of children undergoing congenital heart surgery, it is notably higher than that reported in a previous study from our institution that involved nearly 700 patients who underwent varied surgical procedures. Despite the fact that identical definitions were used in the 2 studies, the prevalence of AKI among patients who underwent surgical procedures of the same complexity as Fontan completion (Risk Adjustment for Congenital Heart Surgery category 3$)^{16}$ was just $21 \%{ }^{4}$ The different prevalence rates suggest that these patients have a predilection, that is not based on technical complexity, toward perioperative AKI.

We hypothesized incorrectly that this susceptibility to AKI would be causally related to the abrupt rise in renal vein pressure that is imposed by Fontan completion, independent of the perfusion pressure across the kidney (renal perfusion pressure). This "renal congestion" pathophysiology (elevated central venous pressure with normal renal perfusion pressure) has been invoked in several experimental and clinical studies of patients with heart failure, ${ }^{17,18}$ but it does not seem to play an important role in the AKI associated with the Fontan procedure. Indeed, our findings are more consistent with recent data suggesting that systemic blood pressure may be more important than central venous pressure in the development of AKI. ${ }^{19}$

We did uncover a potent relationship between renal perfusion pressure and the development of AKI, suggesting a potential loss of autoregulation and/or an inappropriate tolerance of relative hypotension. Traditionally, management of patients recovering from the Fontan procedure has been focused on optimizing cardiac output by volume expansion, minimizing intrathoracic pressure, maintaining low-normal systemic vascular resistance, and enhancing contractility. ${ }^{20-22}$ The results of this study additionally suggest a potential role for the tailoring of inotropic management, to support renal perfusion pressure in the early postoperative period. One may hypothesize, for example, that reduction in milrinone dose and/or addition of a pure vasoconstrictor might increase MAP while decreasing inotrope score, and thereby potentially reduce the risk of AKI. The possible benefits of such a strategy should be investigated in future studies.

The mechanisms by which renal impairment prolongs hospitalization in this patient population are not clear, but fluid homeostasis is well recognized to be a prime determinant of short-term Fontan outcomes. It can be theorized that the AKI-induced inability to effectively excrete water for several days raises filling pressures and prolongs both mechanical ventilation time and duration of pleural effusions, both of which contribute to length of stay after Fontan completion.

Although moderate-to-severe AKI affects short-term outcomes after the Fontan procedure, we did not find any association with follow-up renal dysfunction. Moreover, the prevalence of renal dysfunction in this cohort at follow-up was $8 \%$, lower than might be expected given numerous exposures to potential risk factors for chronic renal insufficiency, including multiple cardiopulmonary bypass runs, treatment with nephrotoxic medications, administration of intravenous iodinated contrast, and chronic cyanosis. The effects of AKI on long-term renal function are not clear in any pediatric population and will require careful follow-up studies of at-risk groups, but our results suggest that renal recovery is to be expected for those children who develop AKI after Fontan procedure surgery.

Patients excluded from the cohort deserve mention. A small number of patients were excluded because of a lack of recent preoperative creatinine; we expect that the effect of excluding them is small in light of the number involved. Our central hypothesis that central venous pressure may be a predictor of AKI led us to exclude patients who did not have venous pressure data; given that Fontan pressure did not turn out to be a risk factor, this exclusion might, as seen in retrospect, reflect a missed opportunity to increase overall study numbers. 
We excluded patients who required early extracorporeal membrane oxygenation support, for 3 reasons. First, being on such support during the period when perioperative AKI develops might confound its detection (owing to dilution of creatinine within the circuit). Second, the situation precipitating need for the support presumably had an impact on end-organ oxygen delivery, and could be an overwhelming factor in the development of AKI in such patients. Third, the inflammatory responses to extracorporeal membrane oxygenation and high-volume transfusion might themselves contribute to new or worsening AKI. Given that the number of patients excluded for this reason was small, the impact on study results is likely to be minimal. Finally, the 1 patient who was excluded because of death within 30 days did not in fact have a markedly atypical immediate postoperative course, and as seen in retrospect, could likely have been included. Again, we estimate any effect of this patient on study results to be minimal.

This study has several additional limitations. First, an epidemiologic study cannot prove causality, ie, AKI may not itself prolong hospital length of stay, but it may be statistically associated with an unobserved variable that is biologically causal. We have minimized this risk by using a granular dataset and a moderately large cohort of carefully profiled patients, although other studies will need to confirm our findings. Second, measuring serum creatinine is known to be an imperfect method of detecting changes in renal function; however, it is the only commonly available, clinically relevant method for assessing acute kidney injury at this time. Third, as follow-up creatinine concentrations were not available for all patients (6\% missing), selection bias may have influenced the finding that perioperative AKI was not associated with renal function at follow-up. This possibility is unlikely given that no difference was found in perioperative AKI rates between those who did, versus did not, have a follow-up creatinine measure.

Fourth, the median renal follow-up period for the patients in this cohort was approximately 1 year only, leaving open the possibility that if the patients were followed for a longer period, an association between post-Fontan completion $\mathrm{AKI}$ and renal dysfunction would become evident. This possibility is particularly relevant given the high prevalence of chronic renal insufficiency reported in older patients undergoing the Fontan procedure. Careful longitudinal follow-up of a cohort of patients undergoing Fontan completion is required to assess this possibility. Finally, we cannot conclude that modifying any of the potential risk factors identified in this study will improve patient outcomes.

In conclusion, the clinical implications of this study may be summarized as follows. Acute kidney injury is a common complication of Fontan completion, although most patients suffer only mild injury. Although some of the identified risk factors for AKI are clearly fixed (preoperative pulmonary vascular resistance and degree of atrioventricular valve regurgitation), others might potentially be mitigated. Not surprisingly, minimizing cardiopulmonary bypass time seems to be important for renal protection. In the postoperative phase, lower renal perfusion pressure and higher inotrope score were independently associated with AKI. Although AKI is nearly uniformly transient in these patients, it does affect hospital length of stay, and thus likely increases health resource utilization. Reducing the risk of AKI may be possible through judicious use of inotropes (for example, moderating the dose of milrinone and/or considering pure vasoconstrictors to support MAP and renal perfusion pressure), although the efficacy of any such strategies should be assessed in future prospective studies.

\section{Conflict of Interest Statement}

Authors have nothing to disclose with regard to commercial support.

\section{References}

1. Ishani A, Nelson D, Clothier B, Schult T, Nugent S, Greer N, et al. The magnitude of acute serum creatinine increase after cardiac surgery and the risk of chronic kidney disease, progression of kidney disease, and death. Arch Intern Med. 2011;171:226-33.

2. Lassnigg A, Schmidlin D, Mouhieddine M, Bachmann LM, Druml W, Bauer P, et al. Minimal changes of serum creatinine predict prognosis in patients after cardiothoracic surgery: a prospective cohort study. J Am Soc Nephrol. 2004; 15:1597-605.

3. Blinder JJ, Goldstein SL, Lee VV, Baycroft A, Fraser CD, Nelson D, et al. Congenital heart surgery in infants: effects of acute kidney injury on outcomes. J Thorac Cardiovasc Surg. 2012;143:368-74.

4. Taylor ML, Carmona F, Thiagarajan RR, Westgate L, Ferguson MA, Del Nido PJ, et al. Mild postoperative acute kidney injury and outcomes after surgery for congenital heart disease. J Thorac Cardiovasc Surg. 2013;146:146-52.

5. Li S, Krawczeski CD, Zappitelli M, Devarajan P, Thiessen-Philbrook H, Coca SG, et al. Incidence, risk factors, and outcomes of acute kidney injury after pediatric cardiac surgery: a prospective multicenter study. Crit Care Med. 2011; 39:1493-9.

6. Zappitelli M, Bernier PL, Saczkowski RS, Tchervenkov CI, Gottesman R, Dancea A, et al. A small post-operative rise in serum creatinine predicts acute kidney injury in children undergoing cardiac surgery. Kidney Int. 2009;76: 885-92.

7. Dimopoulos K, Diller GP, Koltsida E, Pijuan-Domenech A, Papadopoulou SA, Babu-Narayan SV, et al. Prevalence, predictors, and prognostic value of renal dysfunction in adults with congenital heart disease. Circulation. 2008;117: 2320-8.

8. Aydin SI, Seiden HS, Blaufox AD, Parnell VA, Choudhury T, Punnoose A, et al. Acute kidney injury after surgery for congenital heart disease. Ann Thorac Surg. 2012;94:1589-95.

9. Toth R, Breuer T, Cserep Z, Lex D, Fazekas L, Sapi E, et al. Acute kidney injury is associated with higher morbidity and resource utilization in pediatric patients undergoing heart surgery. Ann Thorac Surg. 2012;93: 1984-90.

10. Assenza GE, Graham DA, Landzberg MJ, Valente AM, Singh MN, Bashir A, et al. MELD-XI score and cardiac mortality or transplantation in patients after Fontan surgery. Heart. 2013;99:491-6.

11. Schwartz GJ, Munoz A, Schneider MF, Mak RH, Kaskel F, Warady BA, et al. New equations to estimate GFR in children with CKD. J Am Soc Nephrol. 2009;20:629-37.

12. Heilbron DC, Holliday MA, al-Dahwi A, Kogan BA. Expressing glomerular filtration rate in children. Pediatr Nephrol. 1991;5:5-11.

13. Bradley SM, Simsic JM, McQuinn TC, Habib DM, Shirali GS, Atz AM. Hemodynamic status after the Norwood procedure: a comparison of right 
ventricle-to-pulmonary artery connection versus modified Blalock-Taussig shunt. Ann Thorac Surg. 2004;78:933-41; discussion 933-41.

14. Mehta RL, Kellum JA, Shah SV, Molitoris BA, Ronco C, Warnock DG, et al. Acute Kidney Injury Network: report of an initiative to improve outcomes in acute kidney injury. Crit Care. 2007;11:R31.

15. Akcan-Arikan A, Zappitelli M, Loftis LL, Washburn KK, Jefferson LS, Goldstein SL. Modified RIFLE criteria in critically ill children with acute kidney injury. Kidney Int. 2007;71:1028-35.

16. Jenkins KJ, Gauvreau K, Newburger JW, Spray TL, Moller JH, Iezzoni LI. Consensus-based method for risk adjustment for surgery for congenital heart disease. J Thorac Cardiovasc Surg. 2002;123:110-8.

17. Mullens W, Abrahams Z, Francis GS, Sokos G, Taylor DO, Starling RC, et al. Importance of venous congestion for worsening of renal function in advanced decompensated heart failure. J Am Coll Cardiol. 2009;53:589-96.

18. Testani JM, Khera AV, St John Sutton MG, Keane MG, Wiegers SE, Shannon RP, et al. Effect of right ventricular function and venous congestion on cardiorenal interactions during the treatment of decompensated heart failure. Am J Cardiol. 2010;105:511-6.
19. Dupont M, Mullens W, Finucan M, Taylor DO, Starling RC, Tang WH Determinants of dynamic changes in serum creatinine in acute decompensated heart failure: the importance of blood pressure reduction during treatment. Eur J Heart Fail. 2013;15:433-40.

20. Shekerdemian LS, Bush A, Shore DF, Lincoln C, Redington AN. Cardiopulmonary interactions after Fontan operations: augmentation of cardiac output using negative pressure ventilation. Circulation. 1997;96:3934-42.

21. Costello JM, Dunbar-Masterson C, Allan CK, Gauvreau K, Newburger JW, McGowan FX Jr, et al. Impact of empiric nesiritide or milrinone infusion on early postoperative recovery after Fontan surgery: a randomized, double-blind, placebo-controlled trial. Circ Heart Fail. 2014;7:596-604.

22. Mutsuga M, Quinonez LG, Mackie AS, Norris CM, Marchak BE, Rutledge JM, et al. Fast-track extubation after modified Fontan procedure. J Thorac Cardiovasc Surg. 2012;144:547-52.

Key Words: Pediatrics, congenital heart disease, fontan, acute kidney injury

\section{EDITORIAL COMMENTARY}

\section{Acute kidney injury after Fontan completion: Risk factors and outcomes "The good, the bad, and the ugly"}

Tara Karamlou, MD

See related article on pages 190-7.

\section{THE GOOD...}

The article by Esch and colleagues ${ }^{1}$ in this issue of the Journal is an excellent example of a hypothesis-driven investigation of the factors influencing a clinically important postoperative event, acute kidney injury (AKI), by harnessing the information provided by a unique iatrogenic condition-Fontan physiology, in essence, a "natural experiment" of sorts. Although the results of the study did not prove the original hypothesis, that elevated central venous pressure (evident after Fontan completion) was causal in the pathway of AKI, the data did uncover the

From the Division of Pediatric Cardiac Surgery, University of California, San Francisco, Benioff Children's Hospital, San Francisco, Calif.

Disclosures: Author has nothing to disclose with regard to commercial support. Received for publication April 16, 2015; accepted for publication April 17, 2015. Address for reprints: Tara Karamlou, MD, Division of Pediatric Cardiac Surgery, University of California, San Francisco, Benioff Children's Hospital, Mail Stop 0117, 550 16th St, 5th Floor, San Francisco, CA 94518 (E-mail: tara.karamlou@ ucsf.edu).

J Thorac Cardiovasc Surg 2015;150:197-9

$0022-5223 / \$ 36.00$

Copyright (c) 2015 by The American Association for Thoracic Surgery

http://dx.doi.org/10.1016/j.jtcvs.2015.04.050 equally important finding that systemic hypotension-mediated reduction in renal perfusion pressure was likely the primary driver of post-Fontan AKI.

Recently described and validated definitions of renal dysfunction, including the modified Risk, Injury, Failure, Loss, End-stage renal disease (pRIFLE) score $^{2}$

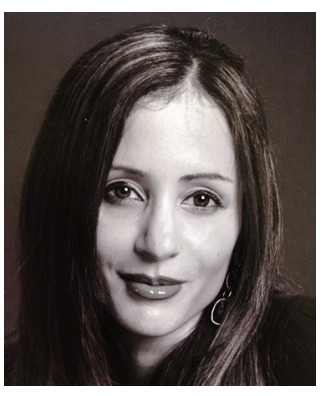
and the Acute Kidney Injury Network (AKIN) criteria, ${ }^{3}$ have made understanding the incidence and impact of AKI more accurate and consistent within pediatric patients and have facilitated stratification into severity quartiles. By using these definitions, the prevalence of AKI has been estimated as high as $56 \%,{ }^{2}$ and therefore the present study investigates a common and important problem among pediatric patients after cardiac surgery. Esch and colleagues ${ }^{1}$ described their population using both the pRIFLE and the AKIN criteria, and they also analyzed whether AKI prevalence or severity stratification would be altered with preferential selection of either criteria. This comparison lends credence to their results and strengthens their article.

Esch and colleagues ${ }^{1}$ selected an ideally suited study population, considering that cyanosis, single ventricle 